\title{
Association of Rheumatoid Arthritis Risk Alleles with Response to Anti-TNF Biologics: Results from the CORRONA Registry and Meta-analysis
}

\author{
Dimitrios A. Pappas, ${ }^{1,6}$ Cheongeun Oh, ${ }^{2}$ Robert M. Plenge, ${ }^{3}$ Joel M. Kremer, ${ }^{4}$ and \\ Jeffrey D. Greenberg 5
}

\begin{abstract}
In this study, we investigated whether genetic variants known to be related with susceptibility to rheumatoid arthritis (RA) are also associated with response to therapy with anti-tumor necrosis factor (anti-TNF) biologics; 233 patients enrolled in the Consortium of Rheumatology Researchers of North America (CORRONA) RA registry were studied. Findings were combined with results from an international collaborative study $(N=1,283)$ in a meta-analysis $(N=1,516)$. Multivariate models investigating the association between single nucleotide polymorphisms (SNPs) and change in RA disease activity were adjusted for age, gender, concomitant methotrexate, and baseline disease activity. In the CORRONA cohort, nominal associations with disease activity improvement were observed for the rs 1980422 SNP of the CD28 gene in multivariate models (coefficient $-0.377, p=0.005$ ) but were not significant after adjustment for multiple comparisons $(q=0.10)$. In the meta-analysis, the only SNP with nominal associations with change in DAS28 was the rs2812378 SNP of the CCL21 gene (coefficient 1.9195, $p=$ $0.0068)$. This association was not significant after adjustment for multiple comparisons $(q=0.143)$. We conclude that the established RA risk alleles studied were not significantly associated with response to anti-TNF biologics in the CORRONA cohort or the meta-analysis.
\end{abstract}

KEY WORDS: rheumatoid arthritis; pharmacogenetics; TNF-alpha inhibitors.

\section{INTRODUCTION}

The discovery and widespread use of anti-tumor necrosis factor (anti-TNF) biologics have improved clinical outcomes for rheumatoid arthritis (RA) patients.

\footnotetext{
${ }^{1}$ Division of Rheumatology, Department of Medicine, New York Presbyterian Hospital, College of Physicians and Surgeons, Columbia University, P\&S Building, Suite 10-455, New York, NY 10032, USA

${ }^{2}$ Biostatistics, Department of Population Health, New York University School of Medicine, 650 First Avenue, \#556, New York, NY 10016, USA

${ }^{3}$ Division of Rheumatology, Immunology and Allergy, Harvard Medical School, New Research Building (NRB), 77 Avenue Louis Pasteur, Boston, MA 02115, USA

${ }^{4}$ Department of Medicine, Albany Medical Center and The Center for Rheumatology, 1367 Washington Ave Ste 101, Albany, NY 12206, USA

${ }^{5}$ Department of Rheumatology, New York University Hospital for Joint Diseases, 301 East 17th Street, Suite 1410, New York, NY 10003, USA

${ }^{6}$ To whom correspondence should be addressed at Division of Rheumatology, Department of Medicine, New York Presbyterian Hospital, College of Physicians and Surgeons, Columbia University, P\&S Building, Suite 10-455, New York, NY 10032, USA. E-mail: dp2544@columbia.edu
}

However, despite achieving unprecedented rates of clinical response, anti-TNF biologics demonstrate failed efficacy in approximately $30 \%$ of patients [1]. It is possible that differential patient response to anti-TNF therapy maypartially - be explained by genetic variability.

In the current study, we sought to further investigate the correlation of genetic variability with response to antiTNF biologics. Single nucleotide polymorphisms (SNPs) previously reported to be associated with susceptibility to development of RA were studied using an independent US cohort and performing a meta-analysis combining results on 20 SNPs from a previously published study of eight European and US cohorts [2].

\section{MATERIAL AND METHODS}

We conducted a longitudinal cohort study of RA patients enrolled in the Consortium of Rheumatology Researchers of North America (CORRONA) registry. A 
detailed description of the CORRONA registry can be found elsewhere [3].

There are 28,635 patients with RA who had been enrolled in CORRONA through June 30, 2011. We identified a subset of patients for whom DNA samples had been previously collected $(N=1,115)$ for the purposes of a genetics sub-study. Subsequently, we isolated those patients with an initiation of adalimumab, etanercept, or infliximab $(N=314)$. All of these patients had a follow-up study visit between 3 and 12 months after initiation of treatment. The rest of the patients $(1,115-314=801)$ did not initiate one of the aforementioned TNF inhibitors and were excluded from the study.

For the purposes of the current investigation, we confined our sample size to biologic naïve $(N=238)$ or first-time switchers of a TNF inhibitor $(N=70)$. The study population was further limited to patients with self-reported white European ancestry $(N=284)$, subsequently to patients who had disease activity assessments at both baseline and follow-up time points and further to patients who at baseline had a DAS28 indicative of active disease (DAS28 $\geq 3.2)(N=233)$.

In addition to analyzing the CORRONA registry data, we performed a meta-analysis including pooled results from an international collaboration of nine cohorts $(N=1,283)$ of patients treated with anti-TNF biologics using published study results [2]. The cohorts included the following: the Autoimmune Biomarkers Collaborative Network $(N=116)$, the Academic Medical Center of the University of Amsterdam $(N=157)$, the Treatment Strategies for Rheumatoid Arthritis (Behandel-strategieen voor Reumatoide Artritis study, $N=126)$, the Biologics in Rheumatoid Arthritis Genetics and Genomics Study Syndicate $(N=81)$, the Brigham Rheumatoid Arthritis Sequential Study $(N=55)$, the Epidemiological Investigation of Rheumatoid Arthritis $(N=291)$, the Immunex Early Rheumatoid Arthritis $(N=218)$, the Karolinska Institute study $(N=163)$, and the Jan van Breemen Institute study $(N=76)$.

Genotyping was performed on 20 established or strongly suggestive non-MHC RA risk alleles for the development of RA [2, 4]. For the CORRONA cohort, genotyping was performed using $5 \mathrm{ng}$ of DNA using the Sequenom MassARRAY iPLEX system at the Broad Institute (Cambridge, MA, USA) as previously described [5]. Individual patients were removed from the cohort if they had $>10 \%$ missing genotype data. In addition, SNPs were removed based on the following criteria: minor allele frequency less than $1 \%$, missing genotype data more than $5 \%$, or significant difference on Hardy-Weinberg equilibrium testing $(p<0.001)$.
Genotype data from the other international collaboration for the meta-analysis were obtained from the published data, with genotyping and imputation methods as described [2]. Disease activity was evaluated using DAS28 (ESR). Disease activity response was assessed in a continuous scale. In order to evaluate disease activity change, we used DAS28 values 3-12 months after initiation of treatment with the anti-TNF agent. If subjects had multiple subsequent registry visits - and multiple DAS28 evaluations-we used the last DAS28 evaluation between 3 and 12 months after the biologic initiation. Imputation of missing ESR values was performed as previously described in order to calculate DAS28 [6].

Univariate predictors of changes in DAS28 were examined in the CORRONA cohort. We first evaluated clinical factors for any association with changes in DAS28, using univariate linear regression. We then tested each SNP for an association with change in DAS28 and also with baseline DAS28 using univariate linear regression. Subsequently, multivariate models examining the association of selected SNPs with response to TNF-a inhibitor therapy were constructed using the CORRONA cohort and the meta-analysis population. Models were developed adjusting for the same covariates as in the international collaboration, including age, gender, concomitant methotrexate, and baseline DAS28. We considered a Bonferronicorrected $p$ value of less than 0.05 as statistically significant, which, in our study of 20 SNPs, corresponded to $p<0.002$. Based on these raw $p$ values obtained from the analysis, the false discovery rate (FDR)-adjusted $q$ values (FDR $5 \%$ ) were calculated using the $q$ value package [7]. All statistical analyses were performed using $\mathrm{R}$ statistical package (r-project.org).

Results from the CORRONA study and International Collaborative Study Cohorts genome-wide analyses were meta-analyzed. We converted $p$ values to $z$ scores from the two studies and then summed the association evidence where the study is weighted in order to perform a fixed effects meta-analysis. Conventional meta-analysis literature advocates the preferred use of random-effects models (rather than fixed-effects models). For genetic discovery, the main focus is on finding novel associations of loci, not on accurate estimation of the effect size. A random-effects metaanalysis effectively penalizes the test statistic for observed heterogeneity between studies, thus lowering power for discovery. Therefore, in our study, our model was a fixed-effects (and not random-effects) model [8]. In order to evaluate the amount of heterogeneity among 
Rheumatoid Arthritis Risk Alleles and Response to Anti-TNF Agents

Table 1. Baseline Characteristics of Patients in the CORRONA Cohort

\begin{tabular}{lr}
\hline & CORRONA cohort $(N=233)$ \\
\hline Female gender $(N, \%)(N=233)$ & $174(74.7)$ \\
Age, mean $( \pm \mathrm{SD})($ years) $(N=233)$ & $56.9(12.3)$ \\
Disease duration, median, mean $(\mathrm{SD})($ years $)(N=232)$ & $6,9.45(9.6)$ \\
Rheumatoid factor positive $(N, \%)(N=191)$ & $139(72.8)$ \\
Background methotrexate $(N, \%)(N=233)$ & $171(73.4)$ \\
Baseline DAS28 median, mean (SD) $(N=233)$ & $4.9,5.2(1.15)$ \\
Follow-up DAS28 median, mean $(\mathrm{SD})(\mathrm{N}=233)$ & $3.3,3.5(1.4)$ \\
Improvement in DAS28 median, mean $(\mathrm{SD})(N=233)$ & $1.6,1.5(1.6)$ \\
Distribution of anti-TNF agents $(N=233)$ & \\
Adalimumab $(N, \%)$ & $97(41.6)$ \\
Etanercept $(N, \%)$ & $57(24.5)$ \\
Infliximab $(N, \%)$ & $79(34)$ \\
\hline
\end{tabular}

cohorts included in this study the $I^{2}$ inconsistency metric was calculated for 5 of the 20 SNPs investigated.

Power calculations were performed. Our study considered a continuous outcome variable (change in DAS28) under a linear regression model. Assuming a minor allele frequency of $10-40 \%$ based on our data and using $0.0025(0.05 / 20)$ as the cutoff $p$ value to declare an association as significant, we had more than $80 \%$ power to detect an additive association explaining $1 \%$ of trait variance for single SNP analysis. Power analysis was done with Genetic Power Calculator [13].

\section{RESULTS}

The clinical characteristics of the 233 patients in the CORRONA cohort are summarized in Table 1 . In the CORRONA cohort, variables with a univariate association with disease activity improvement at the significance level of 0.1 included baseline DAS28 (coefficient $0.679, p<0.001$ ), number of prior DMARDs (coefficient $-0.194, p=0.007$ ), prior use of anti-TNFs (coefficient $-0.85, p<0.001$ ), and current use of MTX (coefficient 0.43, $p=0.055$ ). Patient age, gender, disease duration, and rheumatoid factor status were not associated with change in DAS28 in the CORRONA cohort.

The univariate association between selected SNPS with baseline DAS28 is shown in Table 2. No statistically significant association was identified between any of the investigated SNPs with baseline disease activity. In multivariate models examining the association of individual SNPs with disease activity improvement, we adjusted for age, sex, concurrent treatment with MTX, and the DAS28 at the start of anti-TNF therapy.
As shown in Table 3, no SNP was found to be significantly associated both at a Bonferroni-corrected $p$ value $<0.002$ and at $q$ value $<0.05$ (FDR-adjusted $q$ value), in either corrected univariate or multivariate analyses. In particular, the rs1980422 SNP in the CD28 gene which showed a significant nominal association with improvement of DAS28 (coefficient -0.0375 , $p=0.005$ ) did not maintain statistical significance after adjustment for multiple comparisons (Table 3 ).

Table 2. Association of RA Risk Alleles with Baseline DAS28 in the CORRONA Cohort

\begin{tabular}{lllrrr}
\hline & & & & \multicolumn{2}{c}{ Baseline DAS28 } \\
\cline { 5 - 6 } Chr & Gene & rs\# & MAF & Beta & $P_{\mathrm{N}}$ value \\
\hline 1 & PTPRC & rs10919563 & 0.151 & -0.146 & 0.341 \\
9 & CCL21 & rs2812378 & 0.370 & 0.129 & 0.229 \\
1 & CD58 & s11586238 & 0.266 & -0.112 & 0.367 \\
20 & CD40 & rs4810485 & 0.216 & -0.018 & 0.883 \\
2 & CTLA4 & rs3087243 & 0.439 & -0.038 & 0.717 \\
2 & STAT4 & rs7574865 & 0.258 & -0.215 & 0.080 \\
9 & TRAF1-C5 & rs3761847 & 0.425 & 0.074 & 0.481 \\
2 & REL & rs13031237 & 0.390 & 0.082 & 0.442 \\
10 & PRKCQ & rs4750316 & 0.170 & 0.187 & 0.163 \\
1 & PTPN22 & rs2476601 & 0.104 & 0.120 & 0.464 \\
10 & IL2RA & rs2104286 & 0.242 & -0.173 & 0.151 \\
6 & TAGAP & rs394581 & 0.250 & 0.151 & 0.219 \\
11 & TRAF6 & rs540386 & 0.124 & 0.086 & 0.601 \\
6 & TNFAIP3 & rs10499194 & 0.260 & 0.117 & 0.357 \\
6 & PRDM1 & rs548234 & 0.340 & -0.041 & 0.728 \\
8 & BLK & rs2736340 & 0.242 & -0.068 & 0.560 \\
4 & IL2-IL21 & rs6822844 & 0.169 & 0.075 & 0.607 \\
22 & IL2RB & rs3218253 & 0.249 & -0.032 & 0.777 \\
6 & TNFAIP3 & rs6920220 & 0.220 & -0.218 & 0.089 \\
2 & CD28 & rs1980422 & 0.268 & 0.089 & 0.498 \\
\hline
\end{tabular}


Pappas, Oh, Plenge, Kremer and Greenberg

Table 3. Association of RA Risk Alleles with Change in DAS28 in the CORRONA Cohort

\begin{tabular}{|c|c|c|c|c|c|c|c|c|c|}
\hline \multirow[b]{2}{*}{$\mathrm{Chr}$} & \multirow[b]{2}{*}{ Gene } & \multirow[b]{2}{*}{$\mathrm{rs} \#$} & \multirow[b]{2}{*}{ MAF } & \multicolumn{3}{|c|}{ Univariate } & \multicolumn{3}{|c|}{ Multivariate $^{\mathrm{a}}$} \\
\hline & & & & Beta & $p$ value & $q^{\mathrm{b}}$ value & Beta & $p$ value & $q^{\mathrm{b}}$ value \\
\hline 1 & PTPRC & rs10919563 & 0.151 & -0.059 & 0.772 & 0.876 & 0.061 & 0.727 & 0.898 \\
\hline 9 & CCL21 & rs 2812378 & 0.370 & 0.237 & 0.100 & 0.575 & 0.163 & 0.197 & 0.563 \\
\hline 1 & CD58 & s11586238 & 0.266 & 0.094 & 0.569 & 0.750 & 0.186 & 0.193 & 0.563 \\
\hline 20 & $\mathrm{CD} 40$ & rs 4810485 & 0.216 & -0.091 & 0.593 & 0.750 & -0.117 & 0.426 & 0.852 \\
\hline 2 & CTLA4 & rs3087243 & 0.439 & 0.220 & 0.115 & 0.575 & 0.220 & 0.067 & 0.563 \\
\hline 2 & STAT4 & rs 7574865 & 0.258 & -0.211 & 0.195 & 0.600 & -0.090 & 0.526 & 0.876 \\
\hline 9 & TRAF1-C5 & rs 3761847 & 0.425 & 0.037 & 0.788 & 0.876 & -0.032 & 0.790 & 0.898 \\
\hline 2 & REL & rs13031237 & 0.390 & -0.128 & 0.368 & 0.750 & -0.176 & 0.149 & 0.563 \\
\hline 10 & PRKCQ & rs4750316 & 0.170 & -0.026 & 0.885 & 0.885 & -0.129 & 0.405 & 0.852 \\
\hline 1 & PTPN22 & rs 2476601 & 0.104 & 0.163 & 0.450 & 0.750 & 0.031 & 0.868 & 0.914 \\
\hline 10 & IL2RA & rs 2104286 & 0.242 & -0.099 & 0.536 & 0.750 & 0.034 & 0.808 & 0.898 \\
\hline 6 & TAGAP & rs394581 & 0.250 & 0.093 & 0.569 & 0.750 & -0.008 & 0.956 & 0.956 \\
\hline 11 & TRAF6 & rs540386 & 0.124 & 0.317 & 0.147 & 0.588 & 0.252 & 0.183 & 0.563 \\
\hline 6 & TNFAIP3 & rs10499194 & 0.260 & 0.325 & 0.057 & 0.570 & 0.248 & 0.094 & 0.563 \\
\hline 6 & PRDM1 & rs548234 & 0.340 & -0.110 & 0.488 & 0.750 & -0.070 & 0.613 & 0.876 \\
\hline 8 & BLK & rs 2736340 & 0.242 & -0.193 & 0.210 & 0.600 & -0.144 & 0.282 & 0.705 \\
\hline 4 & IL2-IL21 & rs6822844 & 0.169 & 0.126 & 0.519 & 0.750 & 0.088 & 0.601 & 0.876 \\
\hline 22 & IL2RB & rs3218253 & 0.249 & 0.030 & 0.844 & 0.885 & 0.052 & 0.695 & 0.898 \\
\hline 6 & TNFAIP3 & rs6920220 & 0.220 & -0.089 & 0.600 & 0.750 & 0.080 & 0.590 & 0.876 \\
\hline 2 & CD28 & rs1980422 & 0.268 & -0.422 & 0.006 & 0.120 & -0.375 & 0.005 & 0.100 \\
\hline
\end{tabular}

${ }^{\text {a }}$ Multivariate model adjusted for gender, age, concurrent methotrexate, and baseline DAS

${ }^{b}$ FDR-adjusted $q$ value

Table 4. Association of RA Risk Alleles with Change in DAS28 in Multivariate Models in the International Collaboration Cohorts and Meta-analysis with the CORRONA Cohort

\begin{tabular}{|c|c|c|c|c|c|c|c|}
\hline \multirow[b]{2}{*}{ Gene } & \multirow[b]{2}{*}{ rs \# } & \multicolumn{3}{|c|}{ International collaboration } & \multicolumn{3}{|c|}{ Meta-analysis (fixed effect) } \\
\hline & & Beta & $p$ value & $q^{\mathrm{a}}$ value & Beta & $p$ value & $q^{\mathrm{a}}$ value \\
\hline PTPRC & rs10919563 & -0.28 & 0.0002 & 0.004 & -1.2629 & $0.0749(0.5705)^{b}$ & 0.262 \\
\hline CCL21 & rs2812378 & 0.11 & 0.05 & 0.525 & 1.9195 & 0.0068 & 0.143 \\
\hline CD58 & rs11586238 & 0.08 & 0.25 & 0.817 & 1.4605 & 0.0394 & 0.262 \\
\hline CD40 & rs4810485 & -0.06 & 0.33 & 0.817 & -1.2958 & 0.0675 & 0.262 \\
\hline STAT4 & rs11889341 & 0.07 & 0.30 & 0.817 & 0.9175 & 0.1952 & 0.586 \\
\hline TRAF1-C5 & rs3761847 & 0.05 & 0.33 & 0.817 & -0.4183 & $0.5553(0.7421)^{\mathrm{b}}$ & 0.765 \\
\hline TNFRSF14 & rs3890745 & 0.05 & 0.39 & 0.817 & -0.8618 & 0.2237 & 0.587 \\
\hline REL & rs13031237 & -0.03 & 0.60 & 0.817 & -0.5452 & 0.4413 & 0.765 \\
\hline PRKCQ & rs4750316 & -0.05 & 0.45 & 0.817 & 0.6821 & 0.3354 & 0.762 \\
\hline PTPN22 & rs2476601 & 0.05 & 0.44 & 0.817 & -0.0016 & 0.9982 & 0.998 \\
\hline IL2RA & rs2104286 & -0.04 & 0.50 & 0.817 & 0.4827 & 0.4954 & 0.765 \\
\hline TRAF6 & rs540386 & -0.02 & 0.81 & 0.895 & 0.3636 & $0.6093(0.6923)^{\mathrm{b}}$ & 0.767 \\
\hline TNFAIP3 & rs13207033 & -0.05 & 0.40 & 0.817 & -0.3892 & 0.5825 & 0.765 \\
\hline PRDM1 & rs548234 & -0.02 & 0.70 & 0.817 & 0.2785 & 0.694 & 0.767 \\
\hline BLK & rs13277113 & -0.01 & 0.86 & 0.903 & 0.6897 & $0.3331(0.5272)^{\mathrm{b}}$ & 0.765 \\
\hline IL2-IL21 & rs6822844 & -0.03 & 0.66 & 0.817 & -0.3044 & 0.6674 & 0.767 \\
\hline IL2RB & rs3218253 & 0.03 & 0.68 & 0.817 & 0.5166 & 0.4657 & 0.765 \\
\hline TNFAIP3 & rs6920220 & 0.03 & 0.67 & 0.817 & -1.0418 & $0.1441(0.3629)^{\mathrm{b}}$ & 0.762 \\
\hline CD28 & rs1980421 & -0.01 & 0.92 & 0.920 & -1.2629 & 0.0749 & 0.262 \\
\hline
\end{tabular}

Multivariate model adjusted for gender, age, concurrent methotrexate, and baseline DAS

${ }^{a}$ FDR-adjusted $q$ value

${ }^{b}$ Random-effect $q$ value incorporating between-study heterogeneity 
Data from the meta-analysis also did not reveal any significant associations between individual SNPs and response to TNF therapy. In particular, the rs2812378 in the CCL21 gene which showed a nominal association with disease activity worsening (coefficient 1.9195, $p=0.0068)$ did not maintain statistical significance after adjusting for multiple comparisons in the metaanalysis results (Table 4).

In order to account for the amount of heterogeneity between the investigated cohorts, the $I^{2}$ inconsistency metric was calculated for the selected SNPs. Inconsistency was moderate to very large $\left(I^{2}=40-90 \%\right)$ for five of them. For these five polymorphisms, random-effects calculations incorporating between-study heterogeneity revealed more conservative $p$ values (Table 4) for the summary effects compared with the fixed effects calculations. In addition, we were not able to replicate the positive correlation of the rs10919563 SNP in the PTPRC gene previously reported by Cui et al. [2] in the patient cohort used for our metaanalysis (Table 4).

\section{DISCUSSION}

Large-scale genetic research of RA risk susceptibility, including meta-analyses of genome-wide association studies, have identified a number of genetic variants that predispose to risk of developing RA $[4,9,10]$. To date, the clinical relevance of the majority of these genetic variants for patients who already have a diagnosis of RA remains unclear. A few recently published studies have examined whether these genetic variants may also correlate with the response to anti-TNF agents $[2,11$, 12], but the results have not been consistent.

In this study, we attempted to investigate whether RA risk alleles are associated with response to treatment with anti-TNF agents. Due to the small sample size of our population, we performed a meta-analysis of our findings using data from one of the largest pharmacogenetic cohorts [2]. None of the SNPs evaluated were associated with a response to treatment with TNF inhibitors either in our independent cohort or in the meta-analysis. In addition, we were not able to replicate the previously published positive correlation of the PTPRC SNP with response to anti-TNF therapy.

It is possible that different alleles contribute to RA pathogenesis than the ones interfering with response to therapy. While our population did not have any apparent major differences - including levels of baseline disease activity - compared to the meta-analysis cohort, it is still possible that unaccountable heterogeneity or bias was introduced in the meta-analysis results explaining the negative PTPRC findings. In addition, it is possible that the power of our study was not high enough to detect moderate effect of particular SNPs. We recognize that this is an important limitation of our study.

Strengths of our study include the large number of patients treated with anti-TNFs in the pooled cohorts and the standardized DAS28 measures across all cohorts. Potential limitations include the possibility that our analysis of change in DAS28 as a continuous variable failed to identify phenotype extremes.

In summary, our study indicates that established RA risk alleles are not associated with response to antiTNF biologics.

\section{REFERENCES}

1. Kievit, W., E.M. Adang, J. Fransen, H.H. Kuper, M.A. van de Laar, T.L. Jansen, C.M. De Gendt, D.J. De Rooij, H.L. Brus, P.C. Van Oijen, and P.C. Van Riel. 2008. The effectiveness and medication costs of three anti-tumour necrosis factor alpha agents in the treatment of rheumatoid arthritis from prospective clinical practice data. Annals of the Rheumatic Diseases 67: 1229-1234.

2. Cui, J., S. Saevarsdottir, B. Thomson, L. Padyukov, A.H. van der Helm-van Mil, J. Nititham, L.B. Hughes, N. de Vries, S. Raychaudhuri, L. Alfredsson, J. Askling, S. Wedrén, B. Ding, C. Guiducci, G.J. Wolbink, J.B. Crusius, I.E. van der Horst-Bruinsma, M. Herenius, M.E. Weinblatt, N.A. Shadick, J. Worthington, F. Batliwalla, M. Kern, A.W. Morgan, A.G. Wilson, J.D. Isaacs, K. Hyrich, M.F. Seldin, L.W. Moreland, T.W. Behrens, C.F. Allaart, L.A. Criswell, T.W. Huizinga, P.P. Tak, S.L. Bridges Jr., R.E. Toes, A. Barton, L. Klareskog, P.K. Gregersen, E.W. Karlson, and R.M. Plenge. 2010. Rheumatoid arthritis risk allele PTPRC is also associated with response to anti-tumor necrosis factor alpha therapy. Arthritis and Rheumatism 62: 1849-1861.

3. Kremer, J.M. 2006. The CORRONA database. Autoimmun Reviews 5: 46-54.

4. Stahl, E.A., S. Raychaudhuri, E.F. Remmers, G. Xie, S. Eyre, B.P. Thomson, Y. Li, F.A. Kurreeman, A. Zhernakova, A. Hinks, C. Guiducci, R. Chen, L. Alfredsson, C.I. Amos, K.G. Ardlie, BIRAC Consortium, A. Barton, J. Bowes, E. Brouwer, N.P. Burtt, J.J. Catanese, J. Coblyn, M.J. Coenen, K.H. Costenbader, L.A. Criswell, J.B. Crusius, J. Cui, P.I. de Bakker, P.L. De Jager, B. Ding, P. Emery, E. Flynn, P. Harrison, L.J. Hocking, T.W. Huizinga, D.L. Kastner, X. Ke, A.T. Lee, X. Liu, P. Martin, A.W. Morgan, L. Padyukov, M.D. Posthumus, T.R. Radstake, D.M. Reid, M. Seielstad, M.F. Seldin, N.A. Shadick, S. Steer, P.P. Tak, W. Thomson, A.H. van der Helm-van Mil, I.E. van der HorstBruinsma, C.E. van der Schoot, P.L. van Riel, M.E. Weinblatt, A.G. Wilson, G.J. Wolbink, B.P. Wordsworth, YEAR Consortium, C. Wijmenga, E.W. Karlson, R.E. Toes, N. de Vries, A.B. Begovich, J. Worthington, K.A. Siminovitch, P.K. Gregersen, L. Klareskog, and R.M. Plenge. 2010. Genome-wide association study meta-analysis identifies seven new rheumatoid arthritis risk loci. Nature Genetics 42: 508-514.

5. Raychaudhuri, S., E.F. Remmers, A.T. Lee, R. Hackett, C. Guiducci, N.P. Burtt, L. Gianniny, B.D. Korman, L. Padyukov, F.A. Kurreeman, M. Chang, J.J. Catanese, B. Ding, S. Wong, A.H. van der 
Helm-van Mil, B.M. Neale, J. Coblyn, J. Cui, P.P. Tak, G.J. Wolbink, J.B. Crusius, I.E. van der Horst-Bruinsma, L.A. Criswell, C.I. Amos, M.F. Seldin, D.L. Kastner, K.G. Ardlie, L. Alfredsson, K.H. Costenbader, D. Altshuler, T.W. Huizinga, N.A. Shadick, M.E. Weinblatt, N. de Vries, J. Worthington, M. Seielstad, R.E. Toes, E.W. Karlson, A.B. Begovich, L. Klareskog, P.K. Gregersen, M.J. Daly, and R.M. Plenge. 2008. Common variants at CD40 and other loci confer risk of rheumatoid arthritis. Nature Genetics 40: $1216-1223$.

6. Bentley, M.J., J.D. Greenberg, and G.W. Reed. 2010. A modified rheumatoid arthritis disease activity score without acute-phase reactants (mDAS28) for epidemiological research. Journal of Rheumatology 37: 1607-1614.

7. Green GH and Diggle PJ. On the operational characteristics of the Benjamini and Hochberg false discovery rate procedure. Stat Appl Genet Mol Biol 2007;6:Article27.

8. Normand, S.L. 1999. Meta-analysis: Formulating, evaluating, combining, and reporting. Statistics in Medicine 18: 321-359.

9. Klareskog, L., A.I. Catrina, and S. Paget. 2009. Rheumatoid arthritis. Lancet 373: 659-672.
10. Plenge, R.M. 2009. Recent progress in rheumatoid arthritis genetics: One step towards improved patient care. Current Opinion in Rheumatology 21: 262-271.

11. Potter, C., K.L. Hyrich, A. Tracey, M. Lunt, D. Plant, D.P. Symmons, W. Thomson, J. Worthington, P. Emery, A.W. Morgan, A.G. Wilson, J. Isaacs, A. Barton, and and the BRAGGSS. 2009. Association of rheumatoid factor and anti-cyclic citrullinated peptide positivity, but not carriage of shared epitope or PTPN22 susceptibility variants, with anti-tumour necrosis factor response in rheumatoid arthritis. Annals of the Rheumatic Diseases 68: 69-74.

12. Tan, R.J., L.J. Gibbons, C. Potter, K.L. Hyrich, A.W. Morgan, A.G. Wilson, J.D. Isaacs, BRAGGSS, and A. Barton. 2010. Investigation of rheumatoid arthritis susceptibility genes identifies association of AFF3 and CD226 variants with response to antitumour necrosis factor treatment. Annals of the Rheumatic Diseases 69: 1029-1035.

13. Purcell, S., S.S. Cherny, and P.C. Sham. 2003. Genetic Power Calculator: Design of linkage and association genetic mapping studies of complex traits. Bioinformatics 19(1): 149-150. 